\title{
Ventromedial Prefrontal Cortex Is Necessary for Normal Associative Inference and Memory Integration
}

\author{
Kelsey N. Spalding, ${ }^{1} \oplus^{\oplus}$ Margaret L. Schlichting, ${ }^{2}$ Dagmar Zeithamova, ${ }^{3}{ }^{\circledR}$ Alison R. Preston, 4 - Daniel Tranel, ${ }^{1,5}$ \\ Melissa C. Duff, ${ }^{6}$ and $\odot$ David E. Warren ${ }^{7,5}$ \\ ${ }^{1}$ Department of Psychological and Brain Sciences, College of Liberal Arts and Sciences, University of Iowa, Iowa City, Iowa, ${ }^{2}$ Department of Psychology, \\ University of Toronto, Toronto, Ontario M5S 3G3, Canada, ${ }^{3}$ Department of Psychology, University of Oregon, Eugene, Oregon, ${ }^{4}$ Departments of Psychology \\ and Neuroscience, The University of Texas at Austin, Austin, Texas, ${ }^{5}$ Department of Neurology, Carver College of Medicine, University of Iowa, Iowa City, \\ Iowa, ${ }^{6}$ Department of Hearing and Speech Sciences, Vanderbilt University Medical Center, Nashville, Tennessee, and ${ }^{7}$ Departments of Neurological \\ Sciences and Psychiatry, University of Nebraska Medical Center, Omaha, Nebraska 68198
}

The ability to flexibly combine existing knowledge in response to novel circumstances is highly adaptive. However, the neural correlates of flexible associative inference are not well characterized. Laboratory tests of associative inference have measured memory for overlapping pairs of studied items (e.g., AB, BC) and for nonstudied pairs with common associates (i.e., AC). Findings from functional neuroimaging and neuropsychology suggest the ventromedial prefrontal cortex (vmPFC) may be necessary for associative inference. Here, we used a neuropsychological approach to test the necessity of vmPFC for successful memory-guided associative inference in humans using an overlapping pairs associative memory task. We predicted that individuals with focal vmPFC damage $(n=5 ; 3 \mathrm{~F}, 2 \mathrm{M})$ would show impaired inferential memory but intact non-inferential memory. Performance was compared with normal comparison participants $(n=$ $10 ; 6 \mathrm{~F}, 4 \mathrm{M})$. Participants studied pairs of visually presented objects including overlapping pairs (AB, BC) and nonoverlapping pairs (XY). Participants later completed a three-alternative forced-choice recognition task for studied pairs (AB, BC, XY) and inference pairs (AC). As predicted, the vmPFC group had intact memory for studied pairs but significantly impaired memory for inferential pairs. These results are consistent with the perspective that the vmPFC is necessary for memory-guided associative inference, indicating that the vmPFC is critical for adaptive abilities that require application of existing knowledge to novel circumstances. Additionally, vmPFC damage was associated with unexpectedly reduced memory for $\mathrm{AB}$ pairs post-inference, which could potentially reflect retroactive interference. Together, these results reinforce an emerging understanding of a role for the vmPFC in brain networks supporting associative memory processes.

Key words: associative inference; lesion; memory integration; memory systems; ventromedial prefrontal cortex; vmPFC

\section{Significance Statement}

We live in a constantly changing environment, so the ability to adapt our knowledge to support understanding of new circumstances is essential. One important adaptive ability is associative inference which allows us to extract shared features from distinct experiences and relate them. For example, if we see a woman holding a baby, and later see a man holding the same baby, then we might infer that the two adults are a couple. Despite the importance of associative inference, the brain systems necessary for this ability are not known. Here, we report that damage to human ventromedial prefrontal cortex (vmPFC) disproportionately impairs associative inference. Our findings show the necessity of the vmPFC for normal associative inference and memory integration.

\section{Introduction}

The ability to flexibly relate existing knowledge to novel contexts often requires inference from multiple memory traces (Cohen

Received Aug. 30, 2017; revised March 6, 2018; accepted March 13, 2018.

Author contributions: K.N.S., M.L.S., D.Z., A.R.P., D.T., M.C.D., and D.E.W. designed research; K.N.S. and D.E.W. performed research; K.N.S. and D.E.W. analyzed data; K.N.S., M.L.S., D.Z., A.R.P., D.T., M.C.D., and D.E.W. wrote the paper.

We thank J. Bruss and M. Knirr for help with this project, and the participating patients, their families, and our healthy comparison participants for facilitating our testing.

The authors declare no competing financial interests. and Eichenbaum, 1993; Eichenbaum and Cohen, 2001). For example, imagine your coworker Sarah has pictures of her baby on her desk. Later, at your office party, you see a man walk in carrying the baby from Sarah's pictures. Because the same baby is now a common feature in two different contexts, you may infer that

Correspondence should be addressed to Dr. David E. Warren, University of Nebraska Medical Center, 988440 Nebraska Medical Center, Omaha, NE 68198. E-mail: david.warren@unmc.edu. DOI:10.1523/JNEUROSCI.2501-17.2018

Copyright $\odot 2018$ the authors $\quad 0270-6474 / 18 / 383767-09 \$ 15.00 / 0$ 
Table 1. Demographic and neuropsychological information for vmPFC participants

\begin{tabular}{|c|c|c|c|c|c|c|c|c|c|c|c|c|c|c|c|c|c|c|c|c|}
\hline \multirow[b]{2}{*}{ ID } & \multirow[b]{2}{*}{ Age } & \multirow[b]{2}{*}{ Sex } & \multirow[b]{2}{*}{ Edu } & \multirow[b]{2}{*}{ Eti } & \multirow[b]{2}{*}{ Chron } & \multicolumn{4}{|l|}{ WAIS } & \multirow[b]{2}{*}{ COWA } & \multicolumn{2}{|l|}{$C F$} & \multirow{2}{*}{$\begin{array}{l}\text { AVLT } \\
\text { 1/5/delay }\end{array}$} & \multicolumn{5}{|l|}{ WMS } & \multicolumn{2}{|c|}{ Lesion Vol } \\
\hline & & & & & & VIQ & Sim & $\operatorname{lnf}$ & Voc & & Raw & Norm & & GMI & Al & $A D$ & ADR & VPA & Total & vmPFC \\
\hline 0318 & 77 & M & 14 & Res & 39 & 142 & 18 & 16 & 16 & 54 & 53 & 0.49 & $10 / 14 / 10$ & 109 & 118 & 114 & 125 & 11 & 76,482 & 0.40 \\
\hline 2025 & 64 & $\mathrm{~F}$ & 16 & Str & 19 & 113 & 12 & 11 & 12 & 50 & 49 & -1.08 & $6 / 13 / 6$ & 114 & 105 & 99 & 130 & 8 & 30,433 & 0.44 \\
\hline 2391 & 71 & $\mathrm{~F}$ & 13 & Res & 13 & 110 & 12 & 12 & 12 & 59 & 49 & 0.01 & $11 / 15 / 14$ & 132 & 120 & 124 & 120 & 14 & 53,719 & 0.56 \\
\hline 3350 & 65 & M & 18 & Res & 11 & 119 & 14 & 12 & 11 & 40 & 39 & -1.92 & $8 / 14 / 13$ & 108 & 117 & 114 & 100 & 13 & 34,082 & 0.50 \\
\hline
\end{tabular}

vmPFC participants were cognitively intact, with little evidence of impaired verbal functions or memory. All participants were right handed. ID, Patient identification; Edu, education in years; Eti, etiology; res, resection, str, stroke, SAH, subarachnoid hemorrhage; Chr, chronicity (years between brain injury and experiment); WAIS, Wechsler Adult Intelligence Scale, Ed 3;VIQ, verbal IQ; Sim, Inf, and Voc, similarities, information, and vocabulary subtests age-corrected scaled scores; COWA, Controlled Oral Word Association task scaled scores; CF, category fluency task (raw score and age-corrected Z-score); AVLT, Rey Auditory Verbal Learning Test (trial 1 performance, trial 5 performance, and 30 min delay performance); WMS, Wechsler Memory Scale-III; GMI, WMS general memory index; AI, WMS auditory immediate index; AD, WMS auditory delay index; ADR, WMS auditory delayed recognition index; VPA; WMS verbal paired associates II recall age-corrected scaled scores; Lesion Vol Total, total lesion volume $\left(\mathrm{mm}^{3}\right)$; vmPFC, proportion of vmPFC gray matter affected by lesion.

the man is Sarah's partner. This inference can be drawn without ever seeing Sarah and the man together because a common feature associates the unique memory representations.

The ability to rapidly form new associations and bind together unique memory representations is highly advantageous, because it supports the generation of novel, derived associations in addition to knowledge gained by direct observation (Schlichting and Preston, 2015). By melding associative relationships from multiple events, we can extract commonalities among distinct experiences and infer which situations require similar behaviors or result in similar outcomes (Wilson et al., 2014; Gershman et al., 2015). This is highly adaptive in our rapidly changing everyday environment. Previous work indicates that associative inference relies in part on the hippocampus and medial temporal lobe (MTL; Zeithamova and Preston, 2010), which support declarative relational memory (Cohen and Eichenbaum, 1993; Eichenbaum and Cohen, 2001). However, recent findings suggest that the MTL may interact with medial prefrontal cortex (mPFC), and more specifically the ventromedial prefrontal cortex (vmPFC), in support of associative inference (DeVito et al., 2010; Koscik and Tranel, 2012; Zeithamova et al., 2012a; Preston and Eichenbaum, 2013; Schlichting and Preston, 2016).

The vmPFC is thought to play a role in extracting regularities across experiences, enabling the formation of flexible, dynamic memories (Kroes and Fernández, 2012; Preston and Eichenbaum, 2013; Wilson et al., 2014; Gershman et al., 2015). Additionally, the vmPFC and hippocampus interact during context learning and conceptual learning: the vmPFC is hypothesized to bias hippocampal retrieval toward relevant memories by highlighting task-relevant features and discounting specific details (Rich and Shapiro, 2009; van Kesteren et al., 2012; Navawongse and Eichenbaum, 2013; Mack et al., 2016; Place et al., 2016). Relevant functional neuroimaging findings have shown increased (v)mPFC activity when incorporating previously acquired knowledge to form schematic memories (van Kesteren et al., 2010a,b, 2013; Kroes and Fernández, 2012; Navawongse and Eichenbaum, 2013; Kumaran et al., 2015), and related neuropsychological research conducted in both human and nonhuman animals has demonstrated the necessity of the (v)mPFC for schematic memory (Euston and McNaughton, 2006; Tse et al., 2007; Warren et al., 2014; Spalding et al., 2015).

Associative inference can be examined through tasks requiring inference of relationships based on discrete episodes with overlapping content (i.e., studying $\mathrm{AB}$ and $\mathrm{BC}$, then inferring AC). Functional neuroimaging studies indicate increased vmPFC activation and functional coupling between the vmPFC and hip- pocampus during encoding of overlapping information (Zeithamova and Preston, 2010; Zeithamova et al., 2012a; Schlichting et al., 2015; Schlichting and Preston, 2016) and increased vmPFC activation during correct retrieval of inferred relationships (Zeithamova and Preston, 2010). Additionally, vmPFC activation during encoding of overlapping pairs was found to predict successful expression of inferential memories (Zeithamova et al., 2012a). However, as functional neuroimaging is correlational, it remains unclear whether the vmPFC is necessary for normal associative inference.

We addressed this question using a neuropsychological approach to evaluate the performance of healthy individuals and patients with vmPFC lesions on a task requiring the integration of information learned across overlapping episodes $(A B, B C)$ to draw associative inferences about novel pairs (AC). We predicted that associative inference would be impaired in patients with vmPFC damage due to a reduced ability to flexibly integrate recent experiences. We expected that this impairment would be specific to memory for inferences (AC) because the vmPFC is not hypothesized to be necessary for non-inferential associative memory $(\mathrm{AB}, \mathrm{BC})$.

\section{Materials and Methods \\ Participants}

Participants with bilateral vmPFC lesions ("vmPFC participants": $n=5$; $2 \mathrm{M}, 3 \mathrm{~F}$ ) were selected from the Iowa Neurological Patient Registry. These individuals have focal, stable brain lesions, and they underwent a comprehensive neuropsychological exam at least 3 months after symptom onset (Table 1). Lesions were verified using magnetic resonance imaging or computerized tomography, and the MAP3 lesion method was used to trace lesions in a template space (Damasio and Frank, 1992; Frank et al., 1997). The locus of maximum lesion overlap was the vmPFC, and all had bilateral vmPFC damage (Fig. 1). Additionally, to be eligible for inclusion, participants were required to have intact declarative memory abilities as confirmed by prior neuropsychological testing (Table 1). Healthy normal comparison participants ["normal comparisons" (NC): $n=10 ; 4 \mathrm{M}, 6 \mathrm{~F}$ ] were recruited from the Iowa City area to match to the vmPFC participants $(2: 1)$ on age, sex, and education (mean years of age $=70$, s.d. $=4 ;$ mean years of education $=16$, s.d. $=2$ ). There were no significant differences on any demographic variable, each $t_{(13)}<1$, each $p>0.4$ This study was approved by the Institutional Review Board at the University of Iowa. All participants provided informed consent in accordance with the Declaration of Helsinki and were remunerated for participating.

\section{Materials}

Stimuli were images of 225 common objects. Object stimuli were drawn from Yassa et al. (2011) and Zeithamova et al. (2012a). From these, 135 

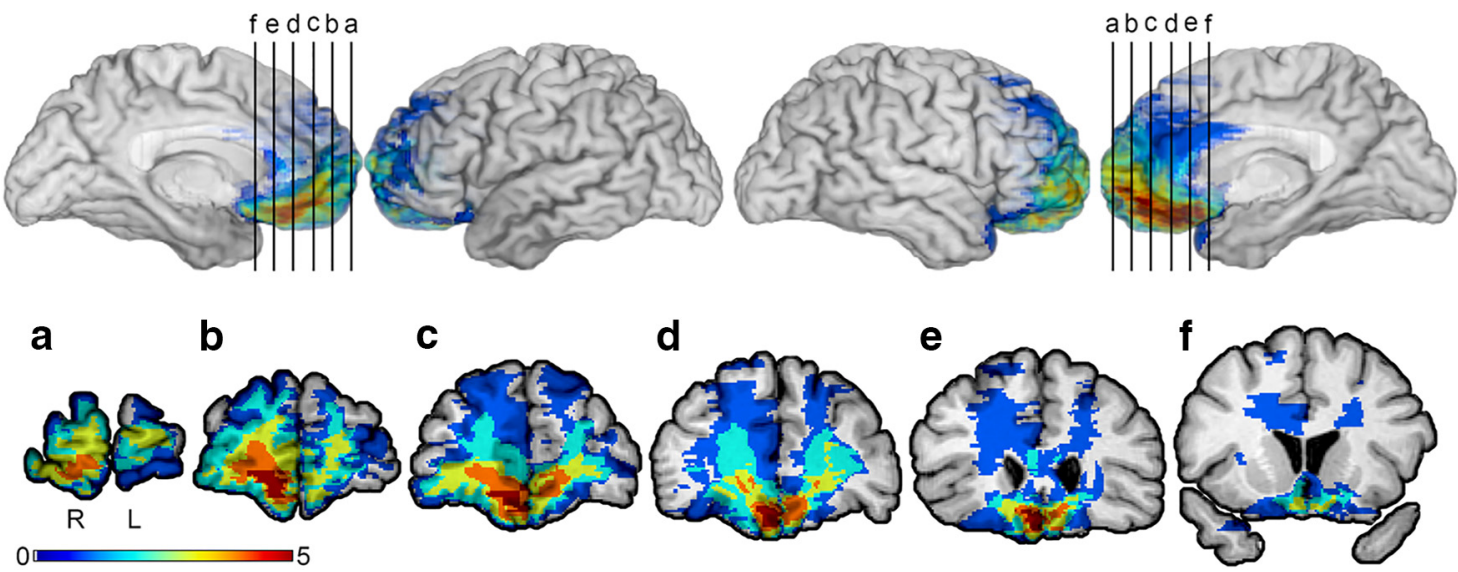

Figure 1. Neuroanatomy of the vmPFC group. Lesion overlap was concentrated in the vmPFC. Hotter colors indicate more cases with overlapping lesions (maximum of 5). Coronal slices correspond to the labels $(\boldsymbol{a}-\boldsymbol{f})$ on the sagittal plane.

items were arranged into 45 triads $(\mathrm{ABC})$. $\mathrm{ABC}$ triads consisted of two studied, overlapping pairs $(\mathrm{AB}$ and $\mathrm{BC})$ as well as a non-studied associative inference pair (AC; Fig. 2). The remaining 90 objects were arranged in 45 nonoverlapping pairs (XY). Four counterbalancing conditions were created to control for the triad/pair assignment of object images and their presentation order.

\section{Experimental design and analyses \\ Procedure}

The task was a modified version of the associative inference paradigm (Preston et al., 2004; Zeithamova and Preston, 2010; Zeithamova et al., 2012a). Stimuli were presented on a computer screen using the MATLAB Psychophysics Toolbox extension (Brainard, 1997; Kleiner et al., 2007). The experiment was separated into four phases: $A B$ Study/Test Phase, BC/XY Study/Test Phase, $A C$ Test Phase, and $\mathrm{AB}_{\text {final }}$ Test Phase (Fig. 2). Participants additionally completed a post-experiment questionnaire.

AB Study/Test Phase. During the AB Study Block, participants viewed 45 pairs of objects. Each pair was presented for $4 \mathrm{~s}$, followed by a fixation cross presented for $1 \mathrm{~s}$. Participants were instructed to remember that the two objects belonged together. They were encouraged to use a narrative or visual imagery to help remember the pairs, but they were not required to make an explicit response during the study phase. After all 45 pairs were presented, participants immediately began the AB Test Block. Participants completed a three-alternative forced-choice (3AFC) recognition task in which they were asked to select which of three objects was paired with a given cue object using a button press. The target alternative was the studied partner matching the cue object, whereas the two lure objects were from other nonmatching studied pairs. Post-response feedback was provided: a green box always appeared around the target object. Additionally, if the participant's response was incorrect, a red " $X$ " appeared over the incorrectly selected object. Participants completed the AB Study/Test Phase two times, with the same pairs presented in a different order each time.

BC/XY Study/Test Phase. During the BC/XY Study Block, participants were instructed that they would be shown new pairs of objects, and they were instructed to remember which new pairs of objects belonged together. Again, participants were encouraged to use a narrative or visual imagery to help remember the pairs, but no explicit response was required during the Study Phase. Participants viewed 45 overlapping BC pairs interleaved with 45 nonoverlapping XY pairs. The XY pairs served as a direct, nonoverlapping comparison for the BC pairs. Participants were not explicitly informed of the overlap of $\mathrm{BC}$ pairs with previously studied AB pairs.

After all pairs were presented, participants immediately began the BC and XY Test Block. Participants completed a 3AFC recognition task in which they were asked to select which object was paired with the cue. BC and XY tests were interleaved, but target and lure items were drawn exclusively from the same BC or XY set. Corrective feedback was pro- vided in the same manner as the $\mathrm{AB}$ Test Phase. Participants completed the BC/XY Study/Test Phase two times, and the same pairs were presented in a different order each time.

AC Test Phase. After the final BC/XY Test Phase, the structure of the inferential associations $(\mathrm{AB}, \mathrm{BC}, \mathrm{AC})$ was explained to participants. They were explicitly told that $\mathrm{A}$ and $\mathrm{C}$ objects paired with the same $\mathrm{B}$ object were indirectly related. Participants then completed a $3 \mathrm{AFC}$ recognition test on the inferential associative $\mathrm{AC}$ items. The format was the same as that of the $\mathrm{AB}$ and $\mathrm{BC} / \mathrm{XY}$ test blocks except that no feedback was provided during the $\mathrm{AC}$ inference test.

$A B_{\text {final }}$ Test Phase. After the AC test phase, participants were once again tested on the $45 \mathrm{AB}$ pairs to assess for retention of the original associations. Participants completed a $3 \mathrm{AFC}$ recognition task in the same format as the previous $\mathrm{AB}$ Test Phase except that no feedback was provided.

Follow-up questionnaire. Finally, participants were asked to complete a questionnaire, which provided an opportunity to disclose any mnemonic strategies they used as well as their observations throughout the experiment (e.g., potential explicit awareness of the overlapping nature of the pairs).

\section{Statistical analyses}

IBM SPSS Statistics for Windows v24.0 was used for statistical analyses. When testing group differences, we chose to use nonparametric inferential statistics (when possible) due to our relatively small sample size. Direct associations, group differences: nonparametric Mann-Whitney $U$ tests were used to investigate group differences in 3AFC recognition performance for direct associations $(\mathrm{AB}, \mathrm{BC}$, and $\mathrm{XY})$. These analyses were repeated for both Test Blocks 1 and 2. Indirect associations, group differences: a nonparametric Mann-Whitney $U$ test was also used to investigate group differences in memory performance for the indirect, inferential associations (AC). Direct versus indirect associations, group differences: To verify that the effect of the vmPFC lesion was specific to inference and was not attributable to any potential differences in direct memory, additional analyses were conducted. First, a mixed effects $2 \times 2$ [group (vmPFC vs NC) $\times$ trial type (direct vs indirect)] ANOVA with repeated measures was performed. This tested for group differences in $3 \mathrm{AFC}$ recognition performance for direct associations (proportion of $\mathrm{AB}, \mathrm{BC}$, and $\mathrm{XY}$ trials correct during their respective Test Block 2) versus indirect associations (proportion of AC trials correct). To further probe this group difference without reliance on parametric assumptions, we also used a nonparametric approach: we calculated a difference score which consisted of the difference in performance on direct (proportion of $\mathrm{AB}, \mathrm{BC}$, and $\mathrm{XY}$ trials correct during Test Block 2) versus indirect associations (proportion of $\mathrm{AC}$ trials correct). Then, we ran a nonparametric Mann-Whitney $U$ test to compare this difference score between groups. Second, group differences in performance on inferential associations were measured using a nonparametric Mann-Whitney $U$ test 
A Stimulus design

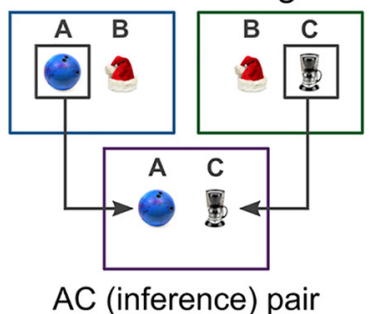

B

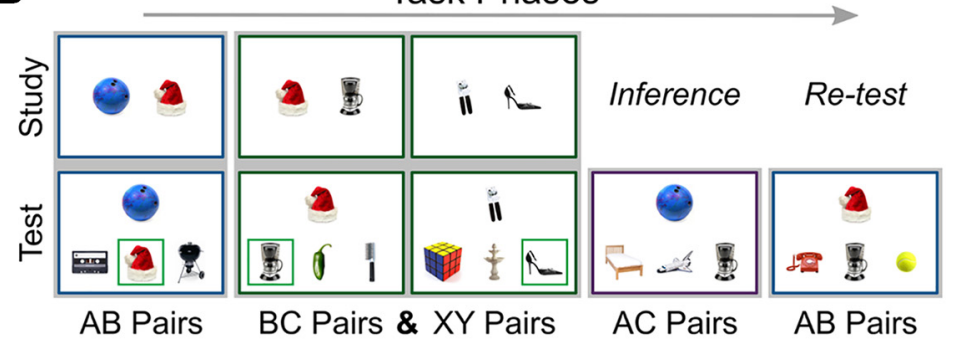

Figure 2. Experimental design. $A$, Example triad. Stimuli were arranged into $45 \mathrm{ABC}$ triads. Triads consisted of two studied, overlapping pairs (AB and $\mathrm{BC}$ ) as well as a non-studied associative inference pair (AC). The A-C labels were not shown to participants, but are included here for clarity. B, Task overview. The task was divided into four phases as depicted above: $A B$ Study/Test Phase, $B C / X Y$ Study/Test Phase, $A C$ Test Phase, and the $A_{\text {final }}$ Test Phase. Participants viewed 45 pairs of items during the $A B$ Study Block and were subsequently tested on these pairs during the $A B$ Test Block. This phase was presented two times. During the BC/XY Study Block, participants viewed 45 overlapping $B C$ pairs interleaved with 45 nonoverlapping XY pairs. They were subsequently tested on these pairs during the $B C / X Y$ Test Block. This phase was repeated two times. During the $A C$ Test Block, participants were tested on the inferential associations. During the $A B_{\text {final }}$ Test Phase, participants were retested on the initially learned $A B$ pairs.

while accounting for memory for the related direct associations. Specifically, this analysis contrasted vmPFC and comparison performance using a conditional measure: mean group proportion correct for only those inferential trials in the AC Test Block in which a participant had previously correctly recalled the corresponding $\mathrm{BC}$ association in $\mathrm{BC}$ Test Block 2 and the $A B$ association during the $\mathrm{AB}_{\text {final }}$ Test Phase $\left(\mathrm{AC}_{\mathrm{alt}}\right)$.

Additionally, exploratory analyses were conducted to investigate potential group differences in interference. Proactive interference: proactive interference was defined as reduced performance on $\mathrm{BC}$ associations relative to XY associations; if this outcome was observed, it could potentially be attributed to prior representations of $B$ from $A B$ pairs. Group differences in proactive interference were investigated with a mixed effects $2 \times 2$ [group $($ vmPFC vs NC) $\times$ trial type $(B C$ vs XY)] ANOVA with repeated measures. This procedure was conducted for both the BC/XY Test Block 1 and Test Block 2, separately. As above, we complemented this parametric analysis with a nonparametric analog: we calculated the difference in performance between $\mathrm{BC}$ and $\mathrm{XY}$ for each subject, and we then used a Mann-Whitney $U$ test was used to compare this difference score between groups. This procedure was completed separately for Test Blocks 1 and 2. $\mathrm{AB}$ retention: potential decline in $\mathrm{AB}$ recognition performance, possibly attributable to retroactive interference from subsequently learned $\mathrm{BC}$ pairs, was measured as the difference in $\mathrm{AB}$ performance between the second $A B$ Test Block (AB2), and the final Test Block $\left(\mathrm{AB}_{\text {final }}\right)$. Group differences in $\mathrm{AB}$ decline were investigated with a mixed effects $2 \times 2$ (group [vmPFC vs $\mathrm{NC}$ ] by trial type [AB2 vs $\left.\mathrm{AB}_{\text {final }}\right]$ ) ANOVA with repeated measures. Once again, we adopted a parallel nonparametric approach: we calculated the difference in performance between $A B 2$ and $A_{\text {final }}$ for each subject, and we then used a MannWhitney $U$ test to compare this difference score between groups. This analysis was followed with a Wilcoxon signed ranks test to compare performance on $\mathrm{AB}_{\text {final }}$ to $\mathrm{AB} 2$ within each group.

Finally, an exploratory analysis was conducted to test whether memory strength for studied information was similarly predictive of associative inference performance for all participants. Specifically, our analysis examined whether non-inference memory performance in the NC group could accurately predict associative inference performance for all participants using the following approach. We fit a linear regression model to data from the $\mathrm{NC}$ group, which estimated the $\mathrm{NC}$ group's associative inference performance (outcome) using memory performance from the non-inference phases (predictors). We then used the fitted model to generate predictions of associative inference performance for the vmPFC group by entering the vmPFC group's non-inference memory performance as predictors. Last, we compared the model predictions of vmPFC performance to the observed vmPFC associative inference performance. Of note, for all non-inference memory phases, the performance of the vmPFC group was always encompassed within the range of normal performance. Thus, model predictions of vmPFC associative inference performance were generated from non-inference performance data in the same range as the model-fit NC non-inference performance data. To ensure that our analysis was rigorous, we implemented two variations of this approach: (A) Memory performance from each phase of testing used as a predictor. Here, we fit a simple single regression model in which the outcome variable (AC phase performance) was predicted using memory performance from one other phase (e.g., $\mathrm{AB} 1, \mathrm{AB} 2, \mathrm{BC} 1$ ) based on data from the NC group. Seven models were fit corresponding to the seven non-inference memory phases. Each model's fit to the NC data was evaluated, and then each model was used to predict performance of the vmPFC group using their respective scores on the predictor variable. The predicted vmPFC AC scores were then compared with the observed vmPFC AC scores. (B) Memory performance across test phases used as a predictor. Here, we addressed the possibility that factors affecting memory performance across multiple phases (e.g., memory ability) might be related to associative inference performance. We began by extracting shared variance information from memory performance in the NC group across all non-inference phases, then used the extracted information to fit a model predicting NC AC performance, and finally predicted vmPFC AC performance using the fitted model. Specifically, we identified shared variance in NC memory performance across noninference phases using principal components analysis (PCA). Seven components were extracted from the seven input variables (i.e., noninference phases) with the first component capturing the dimension of greatest variance in $\mathrm{NC}$ group memory performance across phases, the second (orthogonal) dimension with the next greatest variance, etc. We then fit linear regression models using participant-level scores on one or more components as predictors and $\mathrm{NC}$ group AC performance as the outcome. Seven regression models were fit such that the first model included scores on the first component, the second model included scores on the first and second components, the third model included scores on the first three components, etc. Then, we used each model to predict vmPFC AC performance based on vmPFC memory performance (nb. vmPFC non-inference memory performance was projected into the orthogonalized principal component space generated from the NC memory performance data).

\section{Results}

We found evidence of impairments in associative inference despite intact memory for non-inferential items. As predicted, 3AFC performance on direct associations during both Test Blocks 1 and 2 did not differ significantly between groups for any item type $(\mathrm{AB}, \mathrm{BC}$, and $\mathrm{XY}$ ), all $U \leq 32.50, \mathrm{~d}<0.50, p>0.370$ (Table 2; Fig. $3 A$ ) demonstrating that the vmPFC group was not impaired on associative memory for directly paired items. This is consistent with prior neuropsychological testing demonstrating the vmPFC patients' intact declarative memory abilities (Table 1). However, and consistent with our hypothesis, the vmPFC group did have significantly reduced performance on the indirect, associative inference (AC) trials relative to the NC group: $U=43.00, d=1.39$, $p=0.028$ (Fig. 3A). A significant interaction between group 
Table 2. Group (vmPFC and NC) performance on the 3AFC recognition test for each item type

\begin{tabular}{|c|c|c|c|c|c|c|c|c|c|c|c|}
\hline & \multirow[b]{2}{*}{ Measure } & \multirow[b]{2}{*}{ Identifier } & \multicolumn{2}{|c|}{ Task phase } & \multirow[b]{2}{*}{$\mathrm{BC} 1$} & \multirow[b]{2}{*}{$\mathrm{BC} 2$} & \multirow[b]{2}{*}{$X Y 1$} & \multirow[b]{2}{*}{$X Y 2$} & \multirow[b]{2}{*}{$A C$} & \multirow[b]{2}{*}{$A C_{\text {alt }}$} & \multirow[b]{2}{*}{$A B_{\text {final }}$} \\
\hline & & & AB1 & AB2 & & & & & & & \\
\hline \multirow[t]{5}{*}{ Individuals } & Prop. correct & 0318 & 0.64 & 0.96 & 0.78 & 0.96 & 0.78 & 0.98 & 0.31 & 0.34 & 0.67 \\
\hline & & 2025 & 0.67 & 1.00 & 0.80 & 0.96 & 0.84 & 1.00 & 0.38 & 0.35 & 0.84 \\
\hline & & 2352 & 0.78 & 0.93 & 0.64 & 0.89 & 0.64 & 0.93 & 0.51 & 0.55 & 0.84 \\
\hline & & 2391 & 0.93 & 0.98 & 0.62 & 0.98 & 0.82 & 0.98 & 0.78 & 0.79 & 0.87 \\
\hline & & 3350 & 0.82 & 0.96 & 0.76 & 0.96 & 0.87 & 0.98 & 0.40 & 0.43 & 0.67 \\
\hline \multirow[t]{7}{*}{ Groups } & Median & vmPFC & 0.78 & 0.96 & 0.76 & 0.96 & 0.82 & 0.98 & 0.40 & 0.43 & 0.84 \\
\hline & & NC & 0.79 & 0.99 & 0.82 & 0.97 & 0.82 & 1.00 & 0.86 & 0.88 & 0.96 \\
\hline & Mean & vmPFC & 0.77 & 0.96 & 0.72 & 0.95 & 0.79 & 0.97 & 0.48 & 0.49 & 0.78 \\
\hline & & NC & 0.81 & 0.98 & 0.78 & 0.96 & 0.80 & 0.95 & 0.76 & 0.76 & 0.91 \\
\hline & SD & vmPFC & 0.12 & 0.03 & 0.08 & 0.03 & 0.09 & 0.02 & 0.18 & 0.18 & 0.10 \\
\hline & & NC & 0.14 & 0.03 & 0.14 & 0.05 & 0.15 & 0.09 & 0.23 & 0.22 & 0.13 \\
\hline & & Values & AB1 & $A B 2$ & $\mathrm{BC} 1$ & $\mathrm{BC} 2$ & $X Y 1$ & $X Y 2$ & $A C$ & $\mathrm{AC}_{\mathrm{alt}}$ & $A B_{\text {final }}$ \\
\hline \multirow[t]{5}{*}{ Group $\Delta$} & & $M-W U$ & 30.0 & 32.5 & 31.5 & 29.0 & 27.5 & 29.5 & 43.0 & 43.0 & 41.5 \\
\hline & & $M-W U, p$ value & 0.594 & 0.371 & 0.440 & 0.679 & 0.786 & 0.594 & 0.028 & 0.028 & 0.040 \\
\hline & & Cohen's d & 0.320 & 0.488 & 0.420 & 0.255 & 0.159 & 0.288 & 1.385 & 1.385 & 1.223 \\
\hline & & MD 95\% Cl lower & -0.196 & -0.044 & -0.246 & -0.044 & -0.156 & -0.022 & -0.559 & -0.575 & -0.313 \\
\hline & & MD 95\% Cl upper & 0.113 & 0.018 & 0.116 & 0.046 & 0.134 & 0.088 & -0.062 & -0.031 & -0.003 \\
\hline
\end{tabular}

Mean, $S D$, and median are provided to reflect group performance on the proportion (Prop) of items recognized correctly. Nonparametric Mann-Whitney $U$ tests ( $M$-W U) were used to investigate differences between groups for each item type. Cohen's $d$ is provided as a measure of effect size, and a $95 \%$ confidence interval on the median difference between groups in performance is provided as an additional indicator of statistical significance.
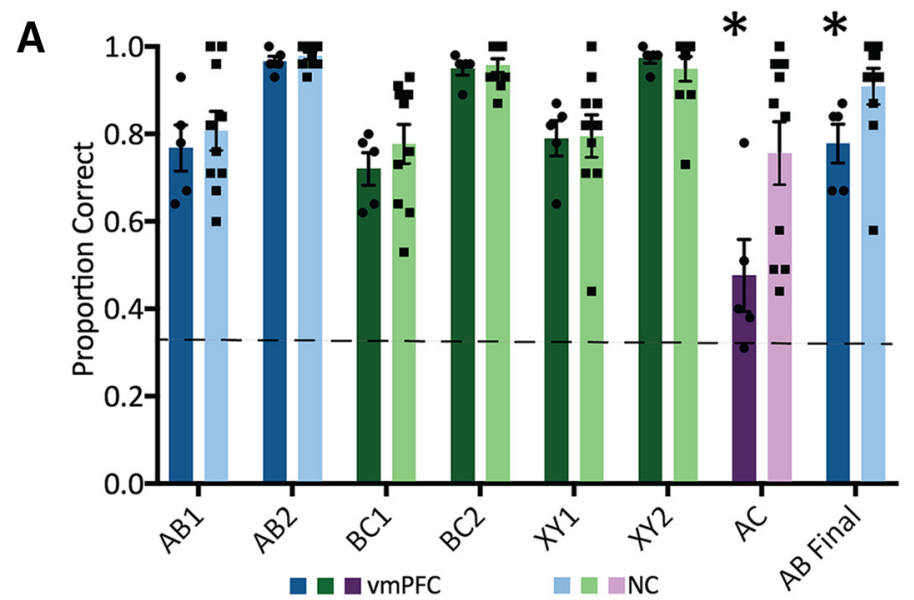

C

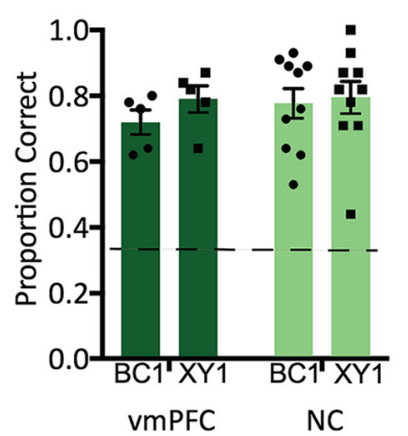

D

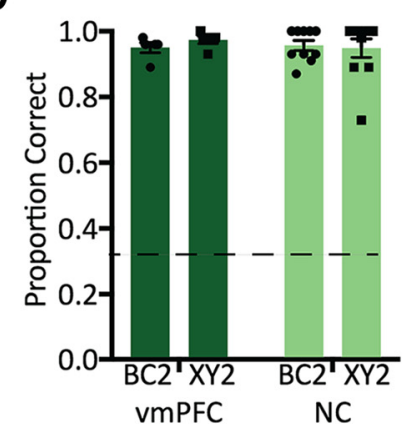

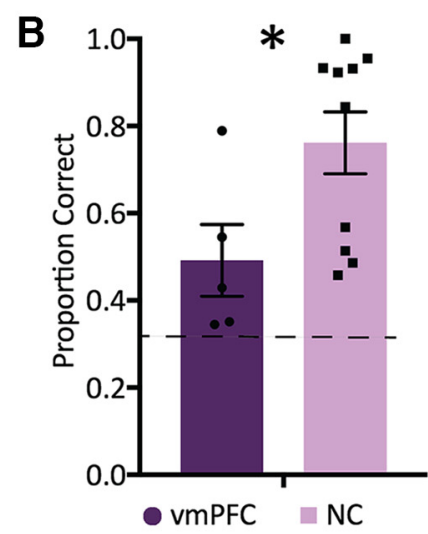

E

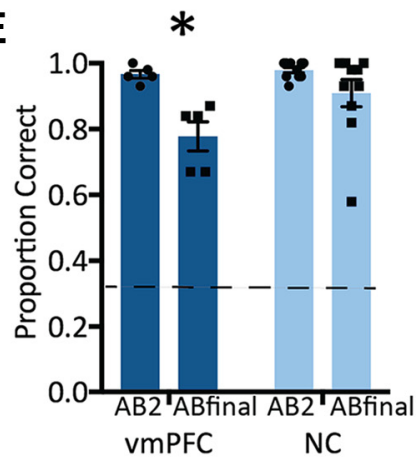

Figure 3. Memory performance. Graphs depict mean performance for each group. Error bars depict SEM. For all panels, the dashed line represents chance ( $1 / 3)$. Bar colors correspond to the phases of the experiment depicted in Figure 2. $\boldsymbol{C}-\boldsymbol{E}$ provide reconfigurations of the data shown in $\boldsymbol{A}$ to illustrate specific contrasts of interest. $\boldsymbol{A}$, Memory performance for all trial types. Results are organized by trial type. The number along the $X$-axis corresponds to the test block (1 or 2). There were no significant differences between groups on memory for the direct associations ( $A B$, $B C, X Y$ ) during Test Blocks 1 or 2 . However, the vmPFC group showed significantly reduced performance in memory for the indirect, inferential associations ( $A C)$. Individuals with vmPFC damage also showed significantly reduced performance when retested on the $A B$ pairs at the end of the experiment ( $A B_{\text {final }}$ Test Phase). $B$, Performance on inferential associations when $A B$ and $B C$ were correct. Performance for inferential associations $(A C)$ on triads in which participants correctly recalled both the $B C$ association at Test 2 and the $A B$ association during the $A B_{\text {final }}$ Test Phase. Under these stringent conditions, the vmPFC group showed significantly impaired performance in memory for the indirect, inferential associations (AC). C, Block 1 proactive interference. A comparison of the performance on $B C$ and $X Y$ associations at Test Block 1. There was not a significant difference in performance on $B C$ versus $X Y$ trials for either group. $D$, Block 2 proactive interference. $A$ comparison of the performance on $B C$ and $X Y$ associations at Test Block 2. There was not a significant difference in performance on $B C$ versus $X Y$ trials for either group. $E$, $A B$ Retention. $A$ comparison of performance during $A B$ Test Block 2 and performance at the completion of the experiment $\left(A B_{\text {final }}\right)$. The vmPFC group showed significantly reduced performance when retested on $A B$ at the completion of the experiment $\left(A B_{\text {final }}\right)$ compared with during $A B$ Test Block 2 . The NC group did not show significant decline in performance. ${ }^{*} p<0.05$. 
(vmPFC vs NC) and trial type (direct vs indirect) in a repeatedmeasures ANOVA confirmed that the observed group difference in associative inference performance was specific to indirect, inferential associations and not direct associations: $F_{(1,13)}=7.10$, $\eta_{\mathrm{p}}^{2}=0.35, p=0.019$. This finding was consistent with a nonparametric Mann-Whitney $U$ test comparing the difference in performance for direct versus indirect associations between groups. This analysis revealed that the disparity between direct and indirect trials was significantly greater for the vmPFC group: $U=$ 43.00, $d=1.39, p=0.028$.

For a more stringent test of whether the observed group difference in associative inference was attributable to differences in memory for the direct pairs, we compared inference (AC) performance between groups for only those triads in which participants correctly recalled both the $\mathrm{AB}\left(\mathrm{AB}_{\text {final }}\right)$ and $\mathrm{BC}(\mathrm{BC} 2)$ pairs. Under this constraint, the vmPFC group continued to show significantly reduced performance on the indirect associative inference (AC) trials relative to the NC group: $U=43.00, d=1.39, p=$ 0.028 (Fig. $3 B$ ). This suggests that the associative inference deficit observed in the vmPFC group during AC testing was not due to impaired memory for the direct pairs.

We probed participants with a post-test questionnaire, and $47 \%$ of all participants noticed the overlapping nature of the $\mathrm{AB}$ and $\mathrm{BC}$ pairs before the explanation given before the $\mathrm{AC}$ test. Specifically, $60 \%$ of patients with vmPFC damage and $40 \%$ of the normal comparison group noticed the overlapping nature of the pairs before explicit instructions. There was not a significant difference between groups, $\chi_{(1)}^{2}=0.54, p=0.464$.

We found no evidence of group differences in proactive interference, but there was some evidence of differences in retention of previous learning. There was not a significant group difference in proactive interference during the BC/XY Test Block 1: $F_{(1,13)}=$ $1.14, \eta_{\mathrm{p}}^{2}=0.081, p=0.304$; or the BC/XY Test Block 2: $F_{(1,13)}=$ 2.01, $\eta_{\mathrm{p}}^{2}=0.134, p=0.180$ (Fig. $3 C, D$ ). These findings were consistent with a nonparametric Mann-Whitney $U$ tests comparing the difference in performance for $\mathrm{BC}$ and $\mathrm{XY}$ between groups. These tests revealed that the difference in performance for $\mathrm{BC}$ and $\mathrm{XY}$ was not significantly different between groups during $\mathrm{BC} / \mathrm{XY}$ Test Block 1: $U=31.50, d=0.42, p=0.440$; or BC/XY Test Block 2: $U=38.50, d=0.94, p=0.099$. Although there was no evidence of proactive interference, there was evidence of a group difference in $\mathrm{AB}$ performance between $\mathrm{AB}$ Study/Test Phase 2 and the $\mathrm{AB}_{\text {final }}$ Test Phase: $F_{(1,13)}=3.46, p=0.086$. This was consistent with a nonparametric Mann-Whitney $U$ test comparing the difference performance during the $\mathrm{AB}_{\text {final }}$ Test Phase and the AB Study/Test Phase 2 between groups: $U=41.5, d=1.22$, $p=0.041$. Follow-up Wilcoxon signed rank tests revealed that both the vmPFC group and the NC group showed significantly reduced performance when retested on the original pairs of items $(\mathrm{AB})$ at the completion of the experiment: $Z=2.03, d=4.33, p=$ 0.042 ; and $Z=1.98, d=1.61, p=0.048$, respectively (Fig. $3 E$ ). This suggests that although there was no evidence of proactive interference, there was some evidence of decline in memory for $\mathrm{AB}$ pairs in the vmPFC group. This could reflect nonspecific decay over time or potential retroactive interference.

We explored the relationship between memory performance during non-inference phases and associative inference performance using a regression-based approach that first modeled NC group data, and then predicted vmPFC group AC-phase performance (see Materials and Methods, Statistical analyses). Memory performance from each phase of testing used as a predictor: as shown in Table 3 (top), although the quality of the fit to the original NC data varied ( $R^{2}$ values between 0.314 and 0.772$)$,
Table 3. Regression fit and predictions of vmPFC group associative inference performance based on data from the NC group

\begin{tabular}{lll}
\hline Model & $R^{2}$ & Diff, $\%$ \\
\hline AB1 & 0.772 & +47.3 \\
AB2 & 0.325 & +44.7 \\
BC1 & 0.745 & +42.3 \\
BC2 & 0.642 & +53.3 \\
XY1 & 0.314 & +58.0 \\
XY2 & 0.324 & +66.4 \\
AB $_{\text {final }}$ & 0.542 & +23.5 \\
PC1 & 0.685 & +45.6 \\
PC1-2 & 0.845 & +31.3 \\
PC1-3 & 0.845 & +31.9 \\
PC1-4 & 0.874 & +37.9 \\
PC1-5 & 0.923 & +40.2 \\
PC1-6 & 0.979 & +26.3 \\
PC1-7 & 0.980 & +28.2 \\
\hline
\end{tabular}

Across several models for different non-inference task phases (top) or data reductions of non-inference performance (bottom), model fit quality $\left(R^{2}\right)$ varied, but all models overestimated vmPFC group performance. $R^{2}$, Non-adjusted $R^{2}$ (single or multiple as appropriate for each single or multiple regression model); Diff $\%$, the difference between predicted and observed vmPFC group performance expressed as a percentage of observed vmPFC performance.

each of the seven non-inference phase models predicted performance in the vmPFC group to be greater than what was observed (min, 23.5\% greater; $\max , 66.4 \%$ greater). Memory performance across test phases used as a predictor: to the extent that common factors drove NC performance across non-inference phases, PCA of NC data extracted those factors from NC data. As in the single regression analysis described previously, every regression model based on PCA scores predicted vmPFC associative inference to be greater than what was observed ( $\mathrm{min}, 26.3 \%$ greater; $\max , 45.6 \%$ greater). In summary, these two regression-based analyses showed that although features of NC memory performance did accurately predict some variance in $\mathrm{NC}$ associative inference performance, those same features did not accurately predict vmPFC associative inference performance. Instead, they uniformly overestimated vmPFC associative inference performance by $>23 \%$. This is consistent with our hypothesis that vmPFC damage disproportionately disrupts associative inference processes.

\section{Discussion}

We observed that individuals with focal, stable lesions of vmPFC had a disproportionate impairment in a memory-guided associative inference task. These results demonstrate the necessity of vmPFC for associated inference and complement previous findings from functional neuroimaging (Zeithamova and Preston, 2010; Zeithamova et al., 2012a; Schlichting and Preston, 2015; Schlichting et al., 2015), which indicated that the vmPFC is a key contributor to a larger memory network supporting associative inference. Lesion studies conducted in both humans and nonhuman animals suggest that vmPFC damage is associated with deficits in transitive inference which requires the formation of hierarchical associations (DeVito et al., 2010; Koscik and Tranel, 2013). The present results extend these findings by showing that the vmPFC is also necessary for non-hierarchical associative memories. Additionally, previous work has suggested that the vmPFC specifically supports socioemotional inferences and is not necessary for nonemotional inferences (Burin et al., 2014). However, the present work suggests that the vmPFC does provide an important contribution to non-emotional associative inference.

Importantly, the vmPFC group did not show a deficit in memory for non-inferential pairs. This is consistent with their performance on neuropsychological tests of associative and non-associative memory (Table 1) and with prior findings demonstrating that indi- 
viduals with vmPFC damage do not show global memory impairment (Warren et al., 2014; Spalding et al., 2015). Instead, the vmPFC group demonstrated a specific reduction in performance on inferential trials, and this is consistent with the perspective that the vmPFC plays a role in a network of brain regions supporting the integration of relational memories (Rubin et al., 2017). Intriguingly, patients with other patterns of frontal lobe damage, specifically basal forebrain amnesia, have shown a complementary pattern of impaired learning of direct associations combined with normal generalization (Myers et al., 2002, 2008; Moustafa et al., 2010).

The hippocampus is also thought to be critical for associative inference (Warren et al., 2016). However, its necessity for direct associative memory (Cohen and Eichenbaum, 1993; Eichenbaum and Cohen, 2001) complicates the measurement of its contribution to indirect associative inference. Interestingly, studies using measures of associative inference in patients with hippocampal lesions suggest that the hippocampus contributes to memory integration in addition to its necessary role in learning direct associations (Myers et al., 2002, 2008; Pajkert et al., 2017).

Previous work demonstrated increased functional coupling between the $\mathrm{mPFC}$ and hippocampus during the encoding of overlapping information (Zeithamova and Preston, 2010; Zeithamova et al., 2012a; Schlichting and Preston, 2015) which predicted subsequent memory for indirect, inferred pairs (AC; Zeithamova et al., 2012a). These findings are broadly consistent with the present results. However, it should be noted that while some of these investigations focused only on vmPFC (Zeithamova et al., 2012a,b) others broadened their focus to the entire mPFC (Schlichting and Preston, 2015, 2016). Similar results have been found across both types of studies, suggesting that perhaps the more ventral regions of the mPFC are particularly important for associative inference (Schlichting et al., 2015). The vmPFC is connected to the MTL both structurally (Cavada et al., 2000; Saleem et al., 2008) and functionally (Hyman et al., 2010). The vmPFC is also extensively connected to sensory and limbic structures (Damasio et al., 1996). This diverse connectivity supports a role for the vmPFC in the integration of information from cortical and subcortical networks supporting associative memories (Cavada et al., 2000).

One potential process by which the vmPFC may impact associative inference is retrieval-mediated learning, or integrative encoding (Shohamy and Wagner, 2008; Zeithamova et al., 2012a). In integrative encoding, the reactivation of details from previous related events enables individual experiences to be encoded in the context of internally generated memory representations of previous experiences. Previous functional neuroimaging research using multivoxel pattern analysis found that prior, related experience appears to be reactivated during encoding, and this reactivation predicted performance on a test of associative inference (Zeithamova et al., 2012a). Reactivation could potentially allow direct integration of the reactivated experience $(\mathrm{AB})$ with the new information $(\mathrm{BC})$ to form a fully integrated memory representation (ABC; O'Reilly and Rudy, 2001; Shohamy and Wagner, 2008; Zeithamova et al., 2012a). The reactivation-integration hypothesis suggests a selective contribution of the vmPFC in the context of a broader set of memory processes and brain networks.

In theories of memory network function, it has been hypothesized that the hippocampus rapidly binds elements of overlapping events into integrated representations during encoding (Eichenbaum and Cohen, 2001; Ranganath, 2010; Zeithamova and Preston, 2010). The vmPFC may bias hippocampal reactivation toward behaviorally relevant memories, and the hippocampus may then bind current experience to the reactivated content, abstracting away from specific details and leading to an integrated memory (Kroes and Fernández, 2012; van Kesteren et al., 2012; Preston and Eichenbaum, 2013; Schlichting and Preston, 2015; Mack et al., 2016; Place et al., 2016). These integrated representations then may be transferred to the vmPFC for future use (Frankland and Bontempi, 2005; Takehara-Nishiuchi and McNaughton, 2008; Takashima et al., 2009; Zeithamova and Preston, 2010; Zeithamova et al., 2012a). Consistent with a role for the vmPFC and hippocampus in retrieval of previously acquired knowledge, changes in right hippocampal and vmPFC encoding activation have been found to predict subsequent inference over and above learning of direct associations, and this suggests that these regions mediate the integration of present experiences with reactivated memories (Zeithamova et al., 2012a). Interestingly, in a single-trial associative learning paradigm, hippocampal but not vmPFC, encoding activation significantly predicted inference performance (Zeithamova and Preston, 2010). This suggests that initial memory integration via the hippocampus proceeds vmPFC involvement.

The current findings are consistent with hypotheses suggesting that the vmPFC plays a role in schema formation, consolidation, and retrieval (Zeithamova et al., 2008; van Kesteren et al., 2012; Preston and Eichenbaum, 2013). Prior work has highlighted the role of the vmPFC in the integration of new information with remotely acquired semantic knowledge, and has suggested that the vmPFC is necessary for the integration of new information with existing contextual memory representations (schemas; van Kesteren et al., 2010a,b, 2013; Preston and Eichenbaum, 2013). This was supported by work demonstrating that vmPFC damage, as well as the temporary disturbance of processing in the vmPFC using transient magnetic stimulation, is associated with impairments in schematic memory (Warren et al., 2014; Spalding et al., 2015; Berkers et al., 2017). Specifically, research suggests vmPFC damage may be associated with an inability to normally integrate new information with previously acquired schemas (Warren et al., 2014; Spalding et al., 2015). The present results extend earlier findings by showing that the vmPFC is also necessary for integrating specific information and deriving novel associations across recently acquired relational memories.

Although our findings regarding associative inference were consistent with our predictions, we also observed that the vmPFC group demonstrated an unexpected reduction in memory performance for the first set of learned pairs $(\mathrm{AB})$ at the completion of the study $\left(\mathrm{AB}_{\text {final }}\right)$. Because a reduction in memory for these direct associations $(\mathrm{AB})$ could impact performance on indirect, inferential associations (AC), we applied a stringent criterion in a follow-up analysis. This analysis demonstrated that relative to the NC group, the vmPFC group showed impaired performance on inferential associations even when analysis was limited to AC pairs in which participants correctly recognized the corresponding $\mathrm{AB}$ and $\mathrm{BC}$ pairs. Although memory for direct associations likely contributes to performance on tests of indirect, inferential associations, this analysis suggests that vmPFC damage is associated with a disproportionate impairment in associative inference that is not readily attributable to impaired memory for direct associations alone.

We discuss two possible interpretations for the decline in $\mathrm{AB}$ performance observed in the vmPFC group: it could reflect nonspecific delay-related decay of associative memory over time; or it could reflect retroactive interference, indicating that learning $\mathrm{BC}$ associations disrupted memory for the original $\mathrm{AB}$ associations. In the latter case, the implication would be that damage to the vmPFC promotes retroactive interference, with the further implication that vmPFC normally suppresses retroactive in- 
terference. Although this was not predicted a priori, it could be consistent with prior models suggesting the vmPFC contributes to the encoding of schema-congruent information (van Kesteren et al., 2012). In the present study, the ability to link $A B$ with $B C$ in an integrated representation could serve as protection against retroactive interference. However, as this integrative process is thought to be reliant on the vmPFC, patients with vmPFC damage may be less able to support integrated " $\mathrm{ABC}$ " representations. Instead, they may rely on unique $\mathrm{AB}$ and $\mathrm{BC}$ memory representations requiring MTL-mediated encoding of episodic information (van Kesteren et al., 2012). If true, then newly learned BC representations may have competed with previously learned $A B$ representations to a greater extent in the vmPFC group than the NC group, resulting in disproportionate retroactive interference in the vmPFC group. Although the current study strongly suggests a role for the vmPFC in associative inference, our findings could also be interpreted to suggest that similar mechanisms support both associative inference and protection against interference. The present study did not include a set of nonoverlapping pairs studied and tested concurrently with $\mathrm{AB}$, and it is therefore not possible to conclude that the decrease in $\mathrm{AB}$ memory is due to either delay-related decay of $\mathrm{AB}$ memory or retroactive interference. Future work could be explicitly designed to test whether the degree of forgetting observed for the $\mathrm{AB}$ pairs is selective for overlapping versus nonoverlapping information.

Although our findings are statistically robust, the present study had some limitations. As is the case for many neuropsychological investigations, our study had a small sample size. This resulted from including only individuals with stable, focal, bilateral damage to the vmPFC in our lesion group. Also, participants in both groups performed near ceiling on the second learning trial for direct associations ( $\mathrm{AB} 1, \mathrm{BC} 1, \mathrm{XY} 1)$. If there were group differences in memory for direct associations, but the ceiling effects masked these differences, they could be responsible for the observed differences in inference. Although this explanation is unlikely, given that performance in the first learning trial never differed between groups, future work could directly address this issue by measuring AC performance after just one exposure. Although the present study suggests that vmPFC damage leads to reduced associative inference, it is possible that additional factors beyond vmPFC damage can also affect this process. This is illustrated by the relatively poor AC performance of several individuals in the NC group. Further, we unexpectedly observed a decline in $\mathrm{AB}$ performance in the vmPFC group, but the design of the current study was not optimized to discern between two possible mechanisms of this effect (decay vs retroactive interference). Future work might be tailored to examine this effect.

\section{Conclusions}

The vmPFC is hypothesized to play a role in the integration of previous knowledge with current experience. Here, we found the vmPFC to be involved with the integration of recently acquired information to enable the formation of dynamic memories supporting second-order, inferential associations even in the absence of reward or hierarchy. Associative inference is highly advantageous because it allows individuals to use prior knowledge flexibly to guide future behavior (Schlichting and Preston, 2015). Whereas associative inference has long been associated with the hippocampus (Eichenbaum, 2000), the present study shows that the vmPFC is necessary for normal associative inference, even when basic associative memory is intact. Additionally, we found that individuals with vmPFC damage showed a post-inference decline in memory for studied direct associations. Future inves- tigations could investigate whether this finding is related to modulation of retroactive interference. These findings are consistent with a role for the vmPFC in a network of brain regions supporting declarative relational memory (Rubin et al., 2017).

\section{References}

Berkers RM, van der Linden M, de Almeida RF, Müller NC, Bovy L, Dresler M, Morris RG, Fernández G (2017) Transient medial prefrontal perturbation reduces false memory formation. Cortex 88:42-52. CrossRef Medline

Brainard DH (1997) The psychophysics toolbox. Spat Vis 10:433-436. CrossRef Medline

Burin DI, Acion L, Kurczek J, Duff MC, Tranel D, Jorge RE (2014) The role of ventromedial prefrontal cortex in text comprehension inferences: semantic coherence or socio-emotional perspective? Brain Lang 129:58 64. CrossRef Medline

Cavada C, Compañy T, Tejedor J, Cruz-Rizzolo RJ, Reinoso-Suárez F (2000) The anatomical connections of the macaque monkey orbitofrontal cortex: a review. Cereb Cortex 10:220-242. CrossRef Medline

Cohen NJ, Eichenbaum H (1993) Memory, amnesia, and the hippocampal system. Cambridge, MA: MIT.

Damasio AR (1996) The somatic marker hypothesis and the possible functions of the prefrontal cortex. Philos Trans R Soc Lond B Biol Sci 351: 1413-1420. CrossRef Medline

Damasio H, Frank R (1992) Three-dimensional in vivo mapping of brain lesions in humans. Arch Neurol 49:137-143. CrossRef Medline

DeVito LM, Lykken C, Kanter BR, Eichenbaum H (2010) Prefrontal cortex: role in acquisition of overlapping associations and transitive inference. Learn Mem 17:161-167. CrossRef Medline

Eichenbaum H (2000) A cortical-hippocampal system for declarative memory. Nat Rev Neurosci 1:41-50. CrossRef Medline

Eichenbaum H, Cohen NJ (2001) From conditioning to conscious recollection: memory systems of the brain. New York: Oxford UP.

Euston DR, McNaughton BL (2006) Apparent encoding of sequential context in rat medial prefrontal cortex is accounted for by behavioral variability. J Neurosci 26:13143-13155. CrossRef Medline

Frank RJ, Damasio H, Grabowski TJ (1997) Brainvox: an interactive, multimodal visualization and analysis system for neuroanatomical imaging. Neuroimage 5:13-30. CrossRef Medline

Frankland PW, Bontempi B (2005) The organization of recent and remote memories. Nat Rev Neurosci 6:119-130. CrossRef Medline

Gershman SJ, Norman KA, Niv Y (2015) Discovering latent causes in reinforcement learning. Curr Opin Behav Sci 5:43-50. CrossRef

Hyman JM, Zilli EA, Paley AM, Hasselmo ME (2010) Working memory performance correlates with prefrontal-hippocampal theta interactions but not with prefrontal neuron firing rates. Front Integr Neurosci 4:2. CrossRef Medline

Kleiner M, Brainard D, Pelli D, Ingling A, Murray R, Broussard C (2007) What's new in psychtoolbox-3. Perception 36:1. CrossRef Medline

Koscik TR, Tranel D (2012) The human ventromedial prefrontal cortex is critical for transitive inference. J Cogn Neurosci 24:1191-1204. CrossRef Medline

Koscik TR, Tranel D (2013) Abnormal causal attribution leads to advantageous economic decision-making: a neuropsychological approach. J Cogn Neurosci 25:1372-1382. CrossRef Medline

Kroes MC, Fernández G (2012) Dynamic neural systems enable adaptive, flexible memories. Neurosci Biobehav Rev 36:1646-1666. CrossRef Medline

Kumaran D, Warren DE, Tranel D (2015) Damage to the ventromedial prefrontal cortex impairs learning from observed outcomes. Cereb Cortex 25:4504-4518. CrossRef Medline

Mack ML, Love BC, Preston AR (2016) Dynamic updating of hippocampal object representations reflects new conceptual knowledge. Proc Natl Acad Sci U S A 113:13203-13208. CrossRef Medline

Moustafa AA, Keri S, Herzallah MM, Myers CE, Gluck MA (2010) A neural model of hippocampal-striatal interactions in associative learning and transfer generalization in various neurological and psychiatric patients. Brain Cogn 74:132-144. CrossRef Medline

Myers CE, Bryant D, DeLuca J, Gluck MA (2002) Dissociating basal forebrain and medial temporal amnesic syndromes: insights from classical conditioning. Integr Physiol Behav Sci 37:85-102. CrossRef Medline

Myers CE, Hopkins RO, DeLuca J, Moore NB, Wolansky LJ, Sumner JM, Gluck MA (2008) Learning and generalization deficits in patients with memory impairments due to anterior communicating artery aneurysm 
rupture or hypoxic brain injury. Neuropsychology 22:681-686. CrossRef Medline

Navawongse R, Eichenbaum H (2013) Distinct pathways for rule-based retrieval and spatial mapping of memory representations in hippocampal neurons. J Neurosci 33:1002-1013. CrossRef Medline

O’Reilly RC, Rudy JW (2001) Conjunctive representations in learning and memory: principles of cortical and hippocampal function. Psychol Rev 108:311-345. CrossRef Medline

Pajkert A, Finke C, Shing YL, Hoffmann M, Sommer W, Heekeren HR, Ploner CJ (2017) Memory integration in humans with hippocampal lesions. Hippocampus 27:1230-1238. CrossRef Medline

Place R, Farovik A, Brockmann M, Eichenbaum H (2016) Bidirectional prefrontal-hippocampal interactions support context-guided memory. Nat Neurosci 19:992-994. CrossRef Medline

Preston AR, Eichenbaum H (2013) Interplay of hippocampus and prefrontal cortex in memory. Curr Biol 23:R764-R773. CrossRef Medline

Preston AR, Shrager Y, Dudukovic NM, Gabrieli JD (2004) Hippocampal contribution to the novel use of relational information in declarative memory. Hippocampus 14:148-152. CrossRef Medline

Ranganath C (2010) Binding items and contexts: the cognitive neuroscience of episodic memory. Curr Dir Psychol Sci 19:131-137. CrossRef

Rich EL, Shapiro M (2009) Rat prefrontal cortical neurons selectively code strategy switches. J Neurosci 29:7208-7219. CrossRef Medline

Rubin RD, Schwarb H, Lucas HD, Dulas MR, Cohen NJ (2017) Dynamic hippocampal and prefrontal contributions to memory processes and representations blur the boundaries of traditional cognitive domains. Brain Sci 7:E82. CrossRef Medline

Saleem KS, Kondo H, Price JL (2008) Complementary circuits connecting the orbital and medial prefrontal networks with the temporal, insular, and opercular cortex in the macaque monkey. J Comp Neurol 506:659-693. CrossRef Medline

Schlichting ML, Preston AR (2015) Memory integration: neural mechanisms and implications for behavior. Curr Opin Behav Sci 1:1-8. CrossRef Medline

Schlichting ML, Preston AR (2016) Hippocampal-medial prefrontal circuit supports memory updating during learning and post-encoding rest. Neurobiol Learn Mem 134:91-106. CrossRef Medline

Schlichting ML, Mumford JA, Preston AR (2015) Learning-related representational changes reveal dissociable integration and separation signatures in the hippocampus and prefrontal cortex. Nat Commun 6:8151. CrossRef Medline

Shohamy D, Wagner AD (2008) Integrating memories in the human brain: hippocampal-midbrain encoding of overlapping events. Neuron 60:378389. CrossRef Medline

Spalding KN, Jones SH, Duff MC, Tranel D, Warren DE (2015) Investigating the neural correlates of schemas: ventromedial prefrontal cortex is necessary for normal schematic influence on memory. J Neurosci 35: 15746-15751. CrossRef Medline

Takashima A, Nieuwenhuis IL, Jensen O, Talamini LM, Rijpkema M, Fernán- dez G (2009) Shift from hippocampal to neocortical centered retrieval network with consolidation. J Neurosci 29:10087-10093. CrossRef Medline

Takehara-Nishiuchi K, McNaughton BL (2008) Spontaneous changes of neocortical code for associative memory during consolidation. Science 322:960-963. CrossRef Medline

Tse D, Langston RF, Kakeyama M, Bethus I, Spooner PA, Wood ER, Witter MP, Morris RG (2007) Schemas and memory consolidation. Science 316:76-82. CrossRef Medline

van Kesteren MT, Rijpkema M, Ruiter DJ, Fernández G (2010a) Retrieval of associative information congruent with prior knowledge is related to increased medial prefrontal activity and connectivity. J Neurosci 30:1588815894. CrossRef Medline

van Kesteren MT, Fernández G, Norris DG, Hermans EJ (2010b) Persistent schema-dependent hippocampal-neocortical connectivity during memory encoding and postencoding rest in humans. Proc Natl Acad Sci U S A 107:7550-7555. CrossRef Medline

van Kesteren MT, Ruiter DJ, Fernández G, Henson RN (2012) How schema and novelty augment memory formation. Trends Neurosci 35:211-219. CrossRef Medline

van Kesteren MT, Beul SF, Takashima A, Henson RN, Ruiter DJ, Fernández G (2013) Differential roles for medial prefrontal and medial temporal cortices in schema-dependent encoding: from congruent to incongruent. Neuropsychologia 51:2352-2359. CrossRef Medline

Warren DE, Jones SH, Duff MC, Tranel D (2014) False recall is reduced by damage to the ventromedial prefrontal cortex: implications for understanding the neural correlates of schematic memory. J Neurosci 34:76777682. CrossRef Medline

Warren DE, Kurczek J, Duff MC (2016) What relates newspaper, definite, and clothing? An article describing deficits in convergent problem solving and creativity following hippocampal damage. Hippocampus 26:835840. CrossRef Medline

Wilson RC, Takahashi YK, Schoenbaum G, Niv Y (2014) Orbitofrontal cortex as a cognitive map of task space. Neuron 81:267-279. CrossRef Medline

Yassa MA, Lacy JW, Stark SM, Albert MS, Gallagher M, Stark CE (2011) Pattern separation deficits associated with increased hippocampal CA3 and dentate gyrus activity in nondemented older adults. Hippocampus 21:968-979. CrossRef Medline

Zeithamova D, Preston AR (2010) Flexible memories: differential roles for medial temporal lobe and prefrontal cortex in cross-episode binding. J Neurosci 30:14676-14684. CrossRef Medline

Zeithamova D, Maddox WT, Schnyer DM (2008) Dissociable prototype learning systems: evidence from brain imaging and behavior. J Neurosci 28:13194-13201. CrossRef Medline

Zeithamova D, Dominick AL, Preston AR (2012a) Hippocampal and ventral medial prefrontal activation during retrieval-mediated learning supports novel inference. Neuron 75:168-179. CrossRef Medline

Zeithamova D, Schlichting ML, Preston AR (2012b) The hippocampus and inferential reasoning: building memories to navigate future decisions. Front Hum Neurosci 6:70. CrossRef Medline 